\title{
Guidelines on service transition for young people with ADHD
}

By Jessica K. Edwards

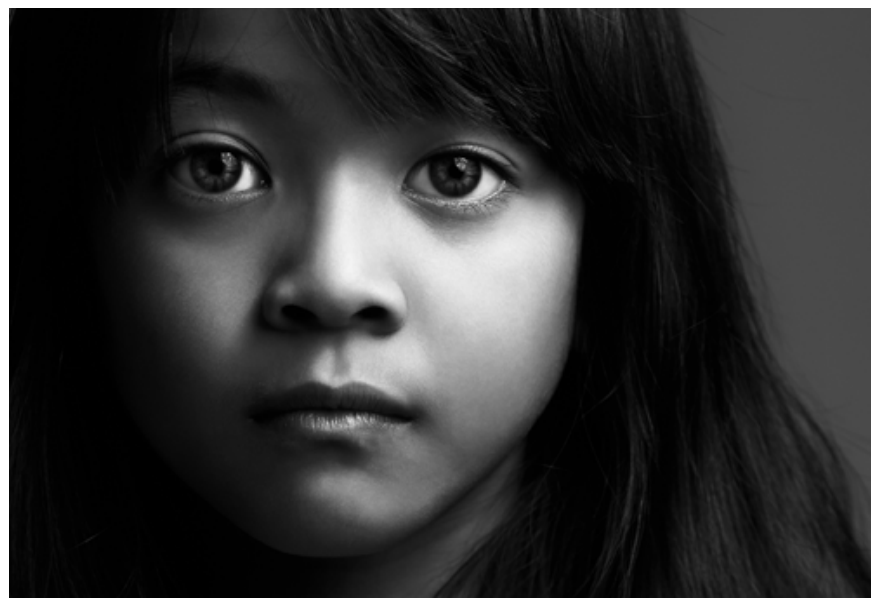

Attention-deficit/hyperactivity disorder (ADHD) affects up to $5 \%$ of children and adolescents in the UK'. Recent research has led to the increasing recognition that ADHD can often be a life span disorder ${ }^{2}$, meaning that a subset of affected children will eventually need to transition to adult services. Unfortunately, much research has highlighted the difficulties experienced by young people in transitioning from children's to adult services ${ }^{3}$. This difficulty is exacerbated by a lack of services and guidelines for young adults with ADHD 4,5 . In 2018, the National Institute of Health and Care Excellence (NICE) updated its guidelines for ADHD (NG87) from earlier versions published in 2008 and 2016. Strikingly, no changes were made with regards to transition recommendations, although the document does refer to a 2016 NICE guideline (NG43) that provides general guidance for health and social care transitions.

Earlier this year, Helen Eke and colleagues from the University of Exeter undertook a Systematic review published in Child and Adolescent Mental Health on the transition from children's to adult services for those living with ADHD in England. They first aimed to identify guidelines for ADHD transition in England and then compared these guidelines with the updated 2018 NICE advice. After searching 10 electronic databases, they identified only 16 documents: most documents based their recommendations for transition on the existing NICE guidelines and only a few independent guidelines were found. Importantly, the NG87 guideline was the only transition guideline that is publically available. With a low rate of successful transitions reported $(\sim 15 \%)^{6}$, the researchers conclude that ADHD-specific guidelines should be made available and accessible to the public to better inform those experiencing transition and make the process as smooth as possible.

\section{Referring to:}

Eke, H., Janssens, A. \& Ford, T. (2019), Review: Transition from children's to adult services: a review of guidelines and protocols for young people with attention deficit hyperactivity disorder in England. Child Adolesc. Ment. Health. 24: 123132 doi: 10.111/camh.12301.

\section{See also:}

https://www.nice.org.uk/guidance/ng87 https://www.nice.org.uk/guidance/ng43 $\mathrm{NG}_{43}$

\section{References:}

${ }^{1}$ Faraone, S.V. et al. (2015), Attention-deficit/ hyperactivity disorder. Nat. Rev. Dis. Primers. 1: 15027. doi: 10.1038/nrdp.2015.20.

${ }^{2}$ Tatlow-Golden, M. et al. (2017), Transitioning from child and adolescent mental health services with attention-deficit hyperactivity disorder in Ireland: Case note review. Early Interv. Psychiatry. 12: 505512. doi: 10.1111/eip.12408.

${ }^{3}$ Singh, S.P. et al. (2008), Transitions of care from child and adolescent mental health services to Adult Mental Health Services (TRACK Study): A study of protocols in Greater London. BMC Health Serv. Res. 8, 135. doi: 10.1186/1472-6963-8-135.

${ }^{4}$ Embrett, M.G. et al. (2016), Effectiveness of health system services and programs for youth to adult transitions in mental health care: A systematic review of academic literature. Adm. Policy Ment. Health. 43, 259-269. doi: 10.1007/s10488-0150638-9.

${ }^{5}$ Seixas, M. et al. (2012), Systematic review of national and international guidelines on attentiondeficit hyperactivity disorder. J. Psychopharmacol. 26, 753-765. doi: 10.1177/0269881111412095.

${ }^{6}$ Singh, S.P. et al. 2015. Transition from child to adult mental health services needs barriers experiences and new models of care. World Psychiatry. 14: 358-361. doi: 10.1002/wps.20266. 